\title{
Estructura social y composición temporal en una colonia de Nyctinomops laticaudatus (Chiroptera: Molossidae)
}

\author{
Social structure and temporal composition in a colony of Nyctinomops laticaudatus \\ (Chiroptera: Molossidae)
}

\author{
Jorge Ortega $^{1 *}$, Beatriz Hernández-Chávez ${ }^{2}$, Areli Rizo-Aguilar ${ }^{3}$ y José Antonio Guerrero ${ }^{3}$ \\ ${ }^{1}$ Laboratorio de Ictiología y Limnología, Posgrado en Ciencias Químico-Biológicas, Escuela Nacional de Ciencias Biológicas, Instituto Politécnico \\ Nacional. Prolongación de Carpio y Plan de Ayala s/n, Col. Sto. Tomas, 11340 México, D.F., México. \\ ${ }^{2}$ Laboratorio de Macroecología, Departamento de Ecología de la Biodiversidad, Instituto de Ecología, Universidad Nacional Autónoma de México. \\ Ciudad Universitaria 04510 México, D.F., México. \\ ${ }^{3}$ Laboratorio de Sistemática y Morfología, Facultad de Ciencias Biológicas, Universidad Autónoma del Estado de Morelos. 62209 Cuernavaca, \\ Morelos, México. \\ *Correspondencia: artibeus2@aol.com
}

\begin{abstract}
Resumen. Poco se conoce acerca de la estructura social de los molósidos en el Neotrópico. En este estudio se observó durante 4 periodos (2006/2007) la distribución espacial y la variación temporal de una colonia de Nyctinomops laticaudatus en el Palacio del Gobernador de la zona arqueológica de Uxmal en Yucatán. Se observaron 66 cavidades ocupadas por grupos mixtos de estos animales. Los grupos mixtos se conservaron todo el año, pero los individuos cambiaron de sitio de percha y de asociación de un grupo a otro. Esta estructura sugiere una organización promiscua. Durante los 2 años de observación sólo se identificaron adultos dentro de las cavidades, mientras que los juveniles y recién nacidos perchaban fuera de éstas. Los periodos de descanso fueron los más comunes en la colonia, pero también se observaron cópulas y visitas de individuos en los sitios de percha. Estos grupos de $N$. laticaudatus se caracterizan por un bajo grado de cohesividad entre sus diferentes miembros.
\end{abstract}

Palabras clave: cavidades de percha, distribución espacial, Nyctinomops laticaudatus, sistema promiscuo, variación temporal.

\begin{abstract}
Little is known about the social structure of the molossid bats in the Neotropics. During 4 observational periods (2006/2007), we studied the spatial distribution and temporal variation of a colony of Nyctinomops laticaudatus inside the Governor's Palace at the Archeological Zone of Uxmal, Yucatán, Mexico. We surveyed 66 roosting cavities consisting of mixed groups of males and females. This particular association (mixed groups) occurred throughout the study, but bats shifted group membership by moving continuously from one group to another. Because of this pattern, we were unable to describe a unique social structure and we proposed a promiscuous system for the species. During the 2 year study, we identified only adult bats inside cavities, while newborns were observed roosting outside cavities in adjacent walls. Resting was the most common activity inside cavities, but visits and copulations were also recorded. In groups of Nyctinomops laticaudatus both sexes are presented and differ with other bats due to their lower degree of cohesiveness.
\end{abstract}

Key words: roosting cavities, spatial distribution, Nyctinomops laticaudatus, promiscuous system, temporal variation.

\section{Introducción}

Los murciélagos son uno de los grupos animales más diversos y gregarios del mundo. Sus formas de agrupación y sistemas sociales están entre los más variados; existen sociedades muy complejas con una fuerte estructura en sus sistemas de apareamiento y también simples parejas

Recibido: 02 junio 2009; aceptado: 23 marzo 2010
(McCracken y Wilkinson, 2000; Kerth, 2008). En los murciélagos gregarios, el potencial de parentesco entre los diferentes miembros del grupo puede definir las interacciones sociales y aumentarlas al grado de tener una selección de grupo basada en relaciones familiares. En grupos sociales de murciélagos estables y cohesivos se han registrado diversas actividades, como la transferencia de información en los sitios de forrajeo (Wilkinson, 1992; Kerth y Reckardt, 2003), el reconocimiento entre 
familiares (Leippert et al., 2000; Ratcliffe et al., 2004; Bohn et al., 2007), la ayuda en el aseo personal (Kerth et al., 2003; Ortega y Maldonado, 2006), los llamados para atraer a las hembras (Davidson y Wilkinson, 2004) o la comunicación mediante llamadas de alerta (Russ et al., 1998; 2004), entre otras. Las interacciones sociales y la conducta dependen del grado de sociabilidad y cohesión de la colonia de murciélagos. Para determinar esta sociabilidad es importante describir la estructura espacial de la agrupación y su variación temporal basada en los movimientos que realizan los individuos a lo largo del tiempo.

En los molósidos y vespertiliónidos, el apareamiento promiscuo es el más común y en muchos sentidos define el arreglo social de los individuos. En este tipo de agrupaciones, la preferencia del macho o de la hembra por algún compañero en particular no está dirigida a determinado miembro, sino que los individuos pueden tener varios compañeros sexuales durante la época reproductiva (McCracken y Wilkinson, 2000). Esta forma de reproducción lleva a encontrar grupos mixtos de individuos sin formar conjuntos de un solo sexo. Por ejemplo, Nyctalus lasiopterus percha en grupos mixtos en las oquedades de los árboles, y en una escala geográfica mayor puede observarse una cohesión de grupo al momento de cambiar el sitio de percha (Popa-Lisseanu et al., 2008).En otros estudios se ha mostrado que las hembras adultas de Eptesicus fuscus están distribuidas en pequeños subgrupos durante el día, pero durante la noche la composición cambia constantemente de miembros y de compañeros sexuales (Willis y Brigham, 2004; Metheny et al., 2008).

En las sociedades de tipo promiscuo, la colonia no se encuentra dividida por sexos, y dependiendo de las condiciones locales los miembros de estos grupos mixtos pueden estar cambiando de sitio de percha (Russo et al., 2005). El tamaño del lugar, el clima o la época del año son condiciones que pueden limitar la membresía en determinados grupos sociales. Los movimientos entre los diferentes sitios de percha crean una composición que puede estar cambiando en el tiempo y en el espacio y permiten interacciones sociales muy diversas entre los diferentes miembros de los grupos.

En muchas especies, una sociedad de tipo promiscuo promueve las asociaciones de individuos al azar y las interacciones sociales son complejas (Leippert et al., 2000).

El murciélago de cola ancha (Nyctinomops laticaudatus) es un molósido de tamaño mediano que se encuentra ampliamente distribuido en las zonas tropicales y subtropicales de América (Ávila-Flores et al., 2002). Este murciélago percha en diversos sitios, cuevas, construcciones humanas o troncos de árboles. Las colonias que habitan las cuevas pueden contener más de 1000 individuos que se encuentran en las grietas que forman las estalactitas en las paredes (Ávila-Flores et al., 2002). En las construcciones humanas, este murciélago percha principalmente bajo las tejas en grupos pequeños (Bowles et al., 1990).

En la Zona Arqueológica de Uxmal en Yucatán se ha registrado una colonia de $N$. laticaudatus que está distribuida en pequeñas unidades sociales dentro de cavidades del edificio denominado Palacio del Gobernador (Malaga y Villa-R., 1956). Estos animales forman una sola entidad cuando salen a forrajear, pero al momento de regresar al sitio de percha se subdividen en pequeños grupos mixtos dentro de las cavidades.

La presencia de estos animales en las cavidades del edificio está registrada desde hace mucho tiempo, y también se ha observado que cambian continuamente de sitio de percha entre las diferentes cavidades, lo cual hace muy dinámico el sistema (Malaga y Villa-R., 1956).

El principal objetivo de este estudio fue determinar la estructura espacial de la colonia de $N$. laticaudatus en las ruinas de Uxmal donde, de acuerdo con datos preliminares, se encontraron grupos mixtos de individuos sin ningún tipo de segregación sexual. El segundo objetivo fue documentar los cambios en la composición de los grupos respecto a la proporción de individuos marcados, originalmente en el grupo, contra la proporción de individuos provenientes de otros grupos. Un tercer objetivo consistió en describir algunas de las conductas sociales de los murciélagos dentro de las diferentes cavidades.

\section{Materiales y métodos}

El estudio se llevó a cabo de mayo de 2006 a noviembre de 2007 en la Zona Arqueológica de Uxmal ( $20^{\circ} 21^{\prime} \mathrm{N}, 89^{\circ}$ 46O), municipio de Santa Elena, en el suroeste de Yucatán. La colonia se encuentra localizada en el edificio del Palacio del Gobernador (Fig. 1), el cual forma parte del complejo de la Zona Arqueológica, y que se caracteriza por tener la presencia continua de una colonia de $N$. laticaudatus (ca. 5 000; Málaga y Villa-R, 1956). La vegetación original de la zona aledaña fue bosque tropical deciduo (Rzedowski, 1978), pero ha sido reemplazado por vegetación secundaria y zonas de cultivo (i. e. maíz, plátano, mangos, etc.).

El Palacio del Gobernador está sobre una pequeña elevación de aproximadamente 6 metros sobre el nivel del suelo. El edificio fue construido entre los siglos VI y $\mathrm{X}$, sobre una plataforma de 12 metros de alto y 100 de largo, y está compuesto por 3 bloques independientes, con 9 cámaras interiores y 11 exteriores. Los grupos de 


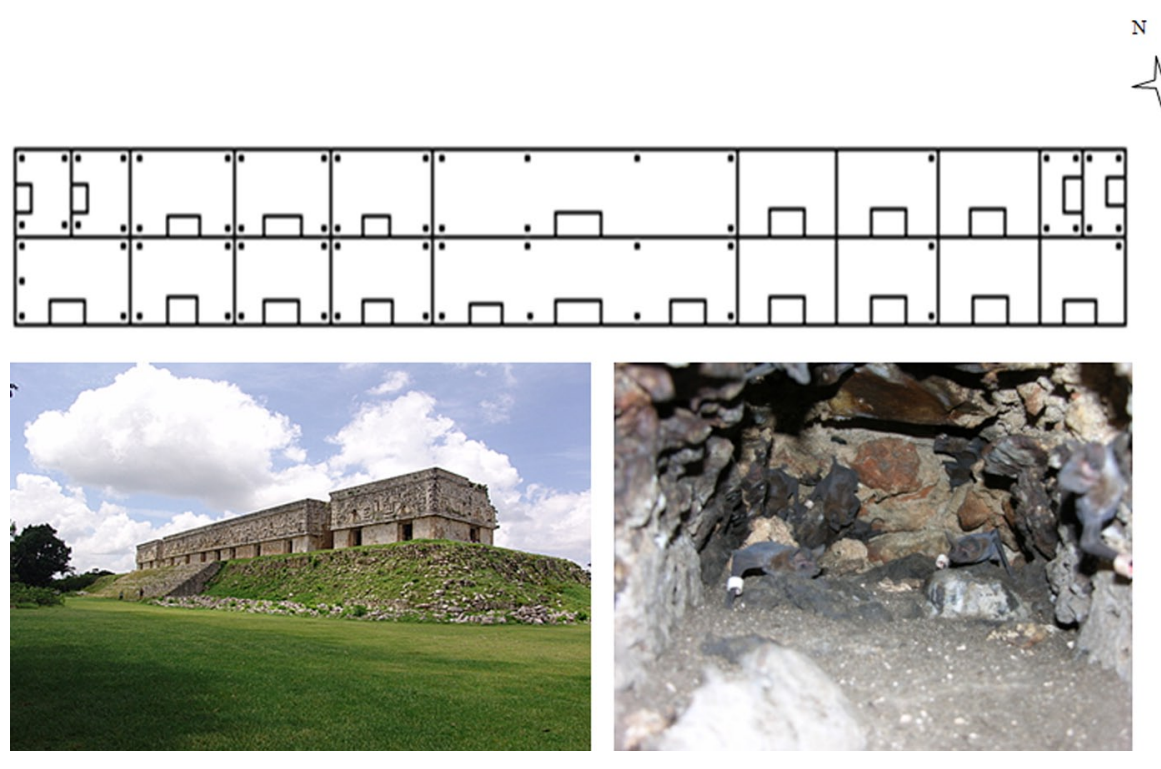

Figura 1. Esquema de distribución de las cavidades dentrodelasdiferentescámaras del Palacio del Gobernador, Zona Arqueológica de Uxmal. Las marcas negras muestran la posición de las cavidades. Fotografías: izquierda, exterior del edificio; derecha, murciélagos marcados, dentro de una cavidad.

$N$. laticaudatus se localizan en pequeñas cavidades, de elaboración humana, que son parte de la construción y que se encuentran en todas las cámaras del edificio a 3 metros del nivel del suelo (Fig. 1). Estas cavidades permanecen oscuras la mayor parte del tiempo, con temperatura constante $\left(28^{\circ} \mathrm{C}\right)$ y humedad relativa superior al $75 \%$.

Las cavidades están localizadas en las esquinas de las cámaras (Fig.1); se registró un total de 66, distribuidas en las diferentes cámaras. La dimensión de cada cavidad es de 30 $\times 30$ centímetros, aproximadamente, con una profundidad promedio de 70 centímetros. En todas las cámaras se registró la presencia de $N$. laticaudatus perchando dentro de estas cavidades, así como la de algunos individuos solitarios perchando en los techos de la edificación (ca. 5 metros), los cuales son difíciles de observar y capturar. Dentro de la edificación existen otras especies de murciélagos (i. e. Artibeus jamaicensis, Peropteryx macrotis y Pteronotus parnellii) que no llegan a formar grupos tan grandes como los de la especie de estudio y no perchan dentro de las pequeñas cavidades, sino en el techo.

Los individuos dentro de las cavidades fueron capturados con la mano y con la ayuda de redes de mano. A todos los organismos de una misma cavidad se les colocó un anillo distintivo para su identificación visual. El anillo de plástico (Avinet, Inc., Dryden, NY; XB tamaño/4.0 mm diámetro) se colocó en el antebrazo derecho de los machos y en el antebrazo izquierdo de las hembras. Cada grupo perteneciente a cada una de las cavidades muestreadas se marcó con un color diferente para hacer distintiva la membresía de los individuos. Debido a la disponibilidad de colores de los anillos, únicamente se pudieron muestrear 23 cavidades diferentes con sus respectivos colores distintivos. Después de que los animales fueron pesados (pesola con calibración al $0.1 \mathrm{~g}$ ), medidos (vernier electrónico con una precisión de $0.1 \mathrm{~mm}$ ) y sexados, se liberaron en la misma cavidad donde fueron capturados. No se observaron efectos deletéreos por el uso de anillos en los murciélagos, y observaciones posteriores mostraron que los individuos conservaban el anillo a largo plazo.

Los murciélagos se clasificaron por edad, de acuerdo con el grado de osificación de las epífisis (Anthony, 1988). Los completamente osificados se consideraron adultos; los parcialmente osificados, juveniles, y los que presentaron una cantidad grande de cartílago en las epífisis se clasificaron como recién nacidos. Para los machos, las condiciones reproductivas se registraron por la presencia de testículos abdominales o escrotados; para las hembras, se registró si estaban lactando, preñadas o sin evidencia física reproductiva.

Después de la captura y marcaje de los individuos, cada una de las 66 cavidades fue monitoreada 2 veces al día durante 15 días, en 4 periodos diferentes: mayo y julio de 2006 y enero y noviembre de 2007. Cada monitoreo se inició a las 8:00 y terminó a las 17:00 horas. Cada observación se enfocó en una cavidad en particular, contando los murciélagos dentro de la misma y registrando su identidad por medio del anillo que se les había colocado con anterioridad. La observación, con duración de unos cuantos minutos, consistió en iluminar la cavidad con una lámpara de cabeza y tomar una serie de 10 fotografías con cámara digital (Canon EOS Rebel Digital). Se determinó la composición de los grupos mediante el conteo directo en las fotografías de los individuos marcados. En cada cavidad se registró la membresía de los individuos, la 
composición sexual de los grupos y el tamaño de cada grupo, dependiendo del número de individuos que apareció en la fotografía de cada cavidad. Los individuos sin anillo en el antebrazo no se consideraron para determinar la membresía de grupo ni para identidad sexual, pero sí para precisar el tamaño del conjunto. Los murciélagos que presentaban el color del anillo y que perchaban en la cavidad original se consideraron residentes repetitivos del sitio de percha, sin identificación individual; los murciélagos miembros de otra cavidad presentes en la cavidad observada, se consideraron miembros inconsistentes del grupo.

La estructura espacial y composición temporal de la colonia se documentó con base en la permanencia, o no, de los individuos en su cavidad original (donde fueron originalmente capturados); además, de acuerdo con su presencia/ausencia en las diferentes cavidades durante los periodos de monitoreo. También se documentó el número de individuos "nuevos" provenientes de otra cavidad y que permanecían perchando en la cavidad de observación. Se consideraron cambios totales los realizados por murciélagos originales de una cavidad cuando fueron registrados en otras cavidades; sin identificación individual, sino únicamente siguiendo el color del anillo. Durante el periodo de lactancia, se registró el número de recién nacidos por conteo directo. Para establecer el sexo se manipularon, pero no se marcaron para evitar que esa acción pudiera perjudicarlos.

Finalmente, se estudiaron algunas actividades realizadas por los murciélagos dentro de las cavidades. Para ello, en cada cavidad se tomó un video de 30 minutos con una cámara de luz infrarroja (ATN NVM-14-3P), la cual no molestó a los murciélagos. Estas grabaciones se realizaron $1 \mathrm{vez}$ al día durante los 4 periodos de monitoreo (2006/2007, para 24 de las 66 cavidades; lo que da un total de 1440 horas/video, con cerca del $70 \%$ de buena calidad). El análisis de los videos consistió en medir el tiempo empleado en las actividades (porcentaje de actividad con respecto al tiempo total de grabación). La observación la realizó una sola persona para evitar sesgos en la interpretación de los datos. Los murciélagos aparentaban dormir durante el comportamiento de descanso, y durante el comportamiento activo realizaron movimientos de alerta, vocalizaciones, aseo y cambios en la posición. Con estos videos, también se registraron las entradas y las salidas de los murciélagos. Debido a que en estos videos no hay color, únicamente se anotó el sexo del animal que salía o entraba, de acuerdo con la colocación del anillo en el antebrazo. Al final se correlacionó el número de entradas y salidas de cada uno de los sexos con la época reproductiva y no reproductiva de la colonia.

\section{Resultados}

Tamaño y composición de la colonia. Se colectaron y marcaron 202 individuos de $N$. laticaudatus del Palacio del Gobernador en Uxmal. El $80 \%$ de estos individuos fueron capturados en mayo del 2006, y el resto fueron colectas ocasionales realizadas en las visitas subsecuentes al sitio de estudio. Durante las capturas realizadas al inicio del estudio se advirtió que los grupos estaban compuestos por individuos de ambos sexos, lo cual fue la base de la estructura social observada durante el resto del estudio. La proporción sexual de todos los murciélagos capturados no difirió de la unidad: los machos estuvieron representados por 81 individuos, mientras que las hembras fueron 121 (prueba binomial de 2 colas, $p=0.73$ ). El mismo patrón se encontró al analizar la composición sexual de cada una de las 23 cavidades muestreadas inicialmente $(n=23$ cavidades, prueba binomial de 2 colas, $p=0.925$ ).

Estructura espacial y composición temporal de los grupos. Las 66 cavidades estudiadas mostraron poca consistencia en cuanto a la permanencia de individuos marcados en la cavidad original. La formación de nuevos grupos provenientes de diferentes cavidades fue una actividad común durante los periodos de monitoreo subsecuentes al marcaje. Estas nuevas formaciones incluyeron machos y hembras de manera indistinta y sin ninguna tendencia en la proporción sexual (prueba binomial de 2 colas, $p=$ 0.57 ). En el tercer periodo de observación se advirtió que 2 grupos dejaron de perchar en 2 cavidades diferentes; uno de los grupos estaba compuesto por 8 hembras $/ 6$ machos y el otro, por 11 hembras/9 machos. Posteriormente, los miembros de estos 2 grupos fueron observados perchando en otras cavidades y se les registró como miembros de esos nuevos grupos.

Los grupos dentro de las cavidades estuvieron compuestos por individuos adultos; ningún recién nacido fue documentado dentro de esas cavidades. La estructura social mostró grupos permanentes a lo largo de todos los periodos de observación, con excepción de los 2 que se desintegraron durante el tercer periodo, como previamente se indicó. De acuerdo con su ubicación con respecto a la cavidad y forma de perchar, los murcielagos se clasificaron en individuos internos, los que perchaban dentro de la cavidad en forma compacta, y en individuos externos, los que permanecían en un radio no mayor a 20 centímetros fuera de la cavidad y ya no perchaban en forma compacta, sino que la distancia entre murciélagos generalmente era mayor a 5 centímetros. Tanto dentro como fuera de la cavidad, los individuos cambiaban de posición durante las observaciones. Los murciélagos cambiaron de plaza de dentro hacia fuera y viceversa varias veces al día, pero no se observó que esta actividad fuera exclusiva de alguno 
de los sexos $($ machos $=25.3 \pm 2.15$ [media $\pm E S$ dentro]; machos $=32.7 \pm 6.82$ [media $\pm E S$ fuera] $;$ hembras $=23.19$ \pm 5.29 [media $\pm E S$ dentro]; hembras $=27.9 \pm 4.92$ [media $\pm E S$ fuera]; ANOVA de una vía, $F=18.22$, g.l. $=35, P$ $>0.05$ ). La composición sexual de los grupos a lo largo de los 4 periodos de observación fue similar; no hubo diferencia significativa entre ellos (ANOVA de una vía, $F$ $=13.21$, g.l. $=3,32 ; P>0.05$; Fig. 2 ).

No se encontró un marcado dimorfismo sexual entre los murciélagos capturados. El tamaño del antebrazo y la masa corporal para ambos sexos no mostraron diferencias significativas $(n=202$ individuos; prueba pareada de 2 colas $t$-test $=5.44$, g.l. $=201, P>0.05$ para el largo del antebrazo [machos: $47.8 \mathrm{~mm}$; hembras $46.6 \mathrm{~mm}$; Fig. 3a]; prueba pareada de 2 colas $t$-test $=11.92$, g.l $l=201$, $P>0.05$ para la masa corporal [machos: $12.18 \mathrm{~g}$; hembras 11.75 g; Fig. 3b] ).

La composición de los grupos mostró una considerable variación durante el estudio. El promedio diario de cambios de murciélagos fue de 156 ( \pm 18.92 ES; $n=115$ murciélagos); en un momento del día se les registraba en una cavidad y en otro momento en otra cavidad. Además de mudarse de sitio por medio del vuelo, los murciélagos utilizaron las grietas que separan las piedras para moverse de una cavidad a la contigua. Durante los 4 periodos de observación, se registró cerca del $80 \%$ de los murciélagos marcados ; posiblemente el $20 \%$ restante perdió la marca y por ello no pudieron localizarse.

Condiciones reproductivas. Los 68 machos capturados mostraron testículos escrotados durante la captura de mayo de 2006, y 13 capturados posteriormente (enero de 2007) los mostraron abdominales. Las hembras manifestaron signos de preñez durante la captura de mayo de $2006(n=31)$, y de lactancia durante las observaciones de julio de 2006 $(n=45)$. Durante las observaciones de enero $(q=22 ; \hat{\partial}=$

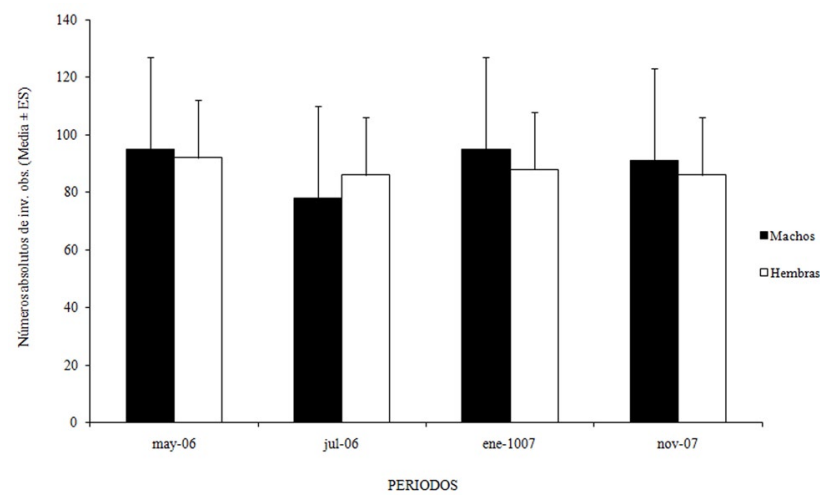

Figura 2. Número de murciélagos adultos. Los números muestran las medias de los 4 periodos $( \pm E S$ ) para ambos sexos en todas las cavidades.
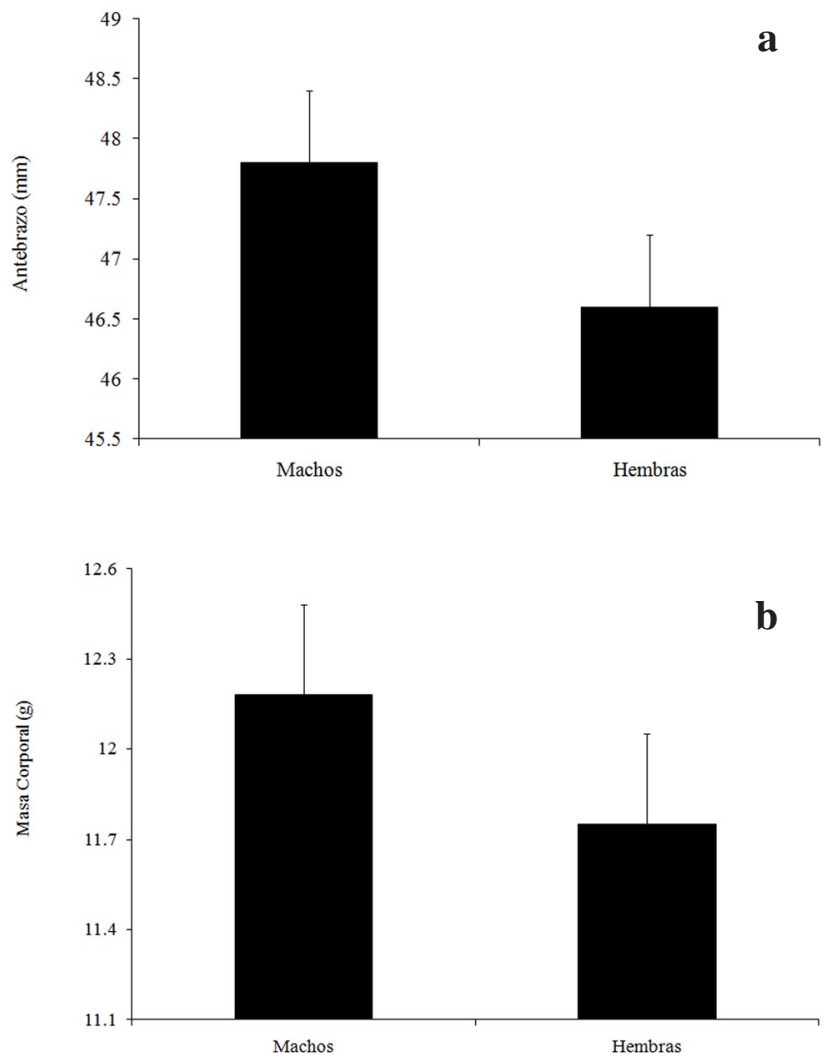

Figura 3. Comparación del largo del antebrazo (a) y de la masa corporal (b), entre machos y hembras adultos de Nyctinomops laticaudatus. No se encontraron diferencias morfológicas evidentes. Las barras de error muestran la desviación estándar.

17) y noviembre de $2007(q=3 ; \hat{\jmath}=26)$, los murciélagos no mostraron señal alguna de actividad reproductiva. Los grupos observados no mostraron diferencia en cuanto a la media de tamaño durante la época reproductiva y la noreproductiva (prueba de $t$-pareada, $t=0.29$, g.l. $=63, P=$ 0.1 ), o en cuanto a la varianza del número de individuos en los grupos (prueba de Bartlett, $F=3.71$, g.l. $=63, P>$ $0.05)$.

El periodo reproductivo previamente registrado para $N$. laticaudatus fue de finales de abril a finales de junio (ÁvilaFlores et al., 2002). Durante estos meses, las observaciones hechas en las grabaciones de video muestran 39 cópulas en 11 diferentes cavidades entre miembros de diferentes grupos: individuos con anillo, e individuos que no habían sido previamente marcados. En 13 casos distintos, los murciélagos adultos ( 8 hembras y 5 machos) copularon inmediatamente después con un individuo diferente. Las cópulas regularmente se registraron a las orillas de las cavidades. Durante la cópula, ambos participantes desplegaban completamente las alas, al tiempo que las agitaban por periodos cortos. 
Se documentó el nacimiento de 56 murciélagos a principios de julio, esto es, el $90 \%$, y el $10 \%$ restante en los últimos días del mes .Los recién nacidos no perchan dentro de las cavidades, sino que las madres los colocan en las paredes internas del edificio, formando una acumulación característica de murciélagos neonatos. Al final del mes se observó que se habían desplazado de su sitio de percha en las paredes hacia el interior de las cavidades. No mostraron desviación en la proporción sexual $(n=56$, prueba binomial de 2 colas, $p=0.84$ ).

Actividades conductuales. El 75\% de las grabaciones muestran que los murciélagos pasaron la totalidad del tiempo en periodo de descanso. Durante el periodo de actividad, los murciélagos generalmente cambian de cavidad. Al momento de arribar a una nueva cavidad, llegan al borde de la misma y comienzan a escalar con las patas y pulgares hasta alcanzar la entrada. La segunda actividad más común fue la de olfatear (9\% del total de observaciones grabadas); la realizan los individuos que están en las cavidades hacia los individuos que recién arriban a las mismas. Finalmente, la tercera actividad en la colonia fue la de autoaseo (6\% del total de las observaciones grabadas).

La frecuencia de llegadas y salidas de las cavidades se muestra en el Cuadro 1. El número de llegadas de las hembras fue similar al de los machos (promedio $[ \pm E S]$ de llegadas observadas para las hembras $74 \pm 4.5$; llegadas observadas para los machos $57 \pm 5.1, n=24$ cavidades en 4 periodos, $t=3.58$, g.l. $=72, P=0.64)$. Las llegadas de las hembras durante la época no reproductiva fueron más que las realizadas durante la época reproductiva (promedio [ \pm $E S$ ] de llegadas de hembras durante la época reproductiva $52 \pm 13.2$ vs. llegadas de hembras durante la época no reproductiva $96 \pm 8.4 n=24$ cavidades, $t=6.92, g . l .=72$, $\mathrm{P}<0.01)$.

Los murciélagos que salían de la cavidad,típicamente volaban alrededor del techo 2 o 3 veces antes de llegar a una nueva cavidad. Tanto los machos como las hembras realizan salidas de las cavidades (promedio [ $\pm E S$ ] de salidas por hembras $68 \pm 7.2$; salidas por machos $60 \pm 5$, $n=24$ cavidades en 4 periodos, $t=8.53$, g.l. $=63, P=0.29$ ). Las hembras salieron de las cavidades con más frecuencia durante la época no reproductiva que en la reproductiva (promedio $[ \pm E S]$ de salidas realizadas durante la época reproductiva $47 \pm 8.24 \mathrm{vs}$. salidas realizadas durante la época no reproductiva $90 \pm 6.4$, Fig. $4 \mathrm{a}$; $n=24$ cavidades, $t=16.32$, g.l. $=63, \mathrm{P}<0.01$; Fig. 4b).

\section{Discusión}

La composición mixta de individuos de ambos sexos para la colonia de $N$. laticaudatus estudiada es similar a la registrada para otras especies de molósidos durante las épocas no reproductivas, como Tadarida brasiliensis (McCracken y Gustin, 1991; Keeley y Keeley, 2004), Mops condylurus (Bouchard, 2001), T. teniotis (Balmori, 2003), Mormopterus planiceps (Krutzsch y Crichton, 2005) y, Molossus pretiosus (Nogueria et al., 2008). Los resultados de este estudio muestran un típico arreglo mixto de individuos en la época reproductiva, sin que exista alguna agregación de tipo harem, como la que se menciona para otras especies de molósidos en época de apareamiento (Balmori, 2003; Keeley y Keeley, 2004). En otras especies de murciélagos se ha registrado la presencia de un macho dominante que controla una gran cantidad de hembras al mismo tiempo (McCracken y Wilkinson, 2000; Kerth, 2008; Muñoz-Romo et al., 2008), pero este no fue el caso para $N$. laticaudatus porque hembra y macho no difieren en cuanto a tamaño; tampoco se observaron despliegues de control por parte de los machos hacia las hembras.

La composición mixta contrasta con otras sociedades de murciélagos que son más complejas, como las de los grupos de harem (Noctilio leporinus [Brooke, 1997], Artibeus jamaicensis [Ortega et al., 2003], A. watsoni [Chaverri et al., 2007], Lophostoma silvicolum [Dechmann et al., 2007] ) y se parece más a sociedades con lazos más libres entre sus diferentes miembros (Kerth, 2008). Los grupos donde existe una panmixia de sexos y no hay evidentes lazos familiares o grupales son típicos de diferentes especies de molósidos (i.e. Tadarida brasiliensis [Keeley y Keeley, 2004], T. teniotis [Balmori, 2003], Mops condylurus [Bouchard, 2001], Mormopterus planiceps [Krutzsch y Crichton, 2005], Molossus pretiosus [Nogueria et al., 2008] ).

Cuadro 1. Media $( \pm E S)$ de arribos y salidas realizados por machos y hembras adultos de Nyctinomops laticaudatus, en las cavidades del Palacio del Gobernador en la Zona Arqueológica de Uxmal, durante los 4 periodos de observación

\begin{tabular}{|c|c|c|c|c|}
\hline Actividad & mayo 2006 & julio 2006 & enero 2007 & noviembre 2007 \\
\hline Arribo de hembras & $43 \pm 6.2$ & $61 \pm 7.0$ & $96 \pm 4.58$ & $97 \pm 5.5$ \\
\hline Salida de hembras & $46 \pm 3.7$ & $49 \pm 2.18$ & $86 \pm 7.7$ & $94 \pm 6.23$ \\
\hline Arribo de machos & $48 \pm 3.2$ & $67 \pm 5.7$ & $56 \pm 4.1$ & $58 \pm 6.24$ \\
\hline Salida de machos & $59 \pm 5.4$ & $72 \pm 3.7$ & $63 \pm 8.4$ & $49 \pm 4.9$ \\
\hline
\end{tabular}



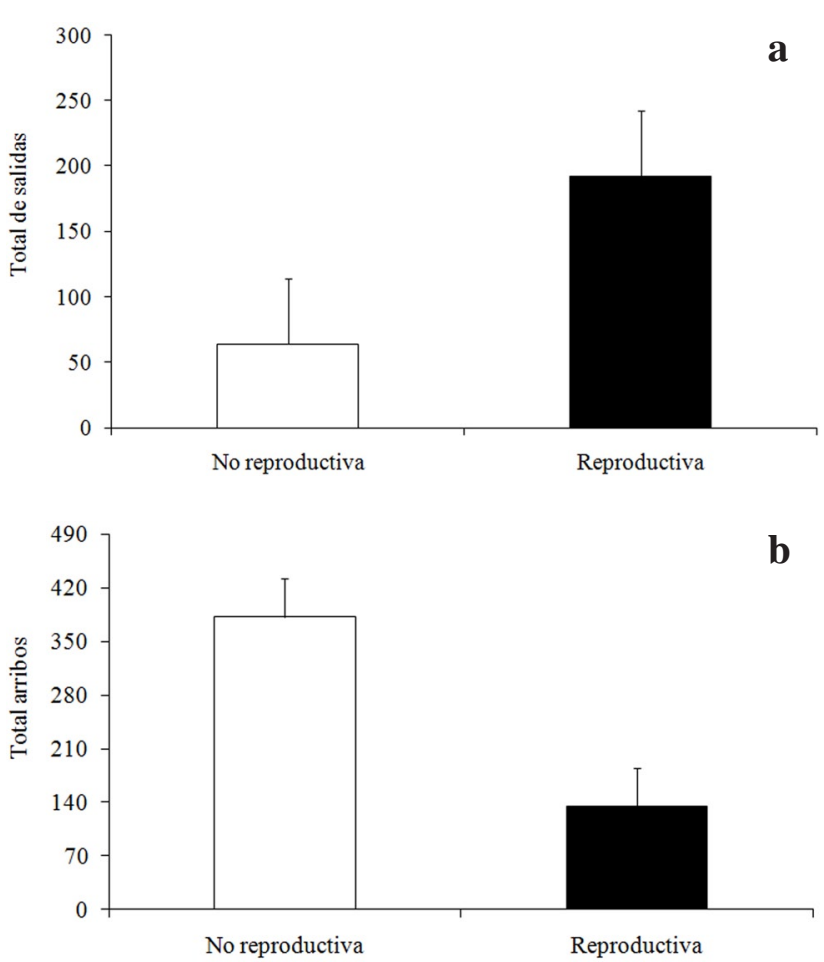

Figura 4. Comparación entre la época reproductiva y la no reproductiva de salidas (a) y arribos (b) realizados por las hembras de Nyctinomops laticaudatus durante los 4 periodos de observación.

La estructura social no está limitada por el número de cavidades, ya que muchas mostraban señales de haber sido ocupadas con anterioridad (signos de excrementos a las orillas) pero que en la actualidad se encuentran vacías. Registramos cambios frecuentes entre los murciélagos de diferentes cavidades, lo que a corto plazo puede tener un costo energético asociado, pero también ciertos beneficios, como evitar los ectoparásitos o escoger sitios con mejores condiciones microambientales (Lewis, 1995).

Los datos recabados durante el estudio sugieren que la colonia está estructurada en grupos mixtos (machos y hembras) dentro de las cavidades. El desarrollo de la sociabilidad y membresía de los grupos está dada principalmente por el grado de asociación que se desarrolla entre los machos y hembras adultos (Kerth, 2008). La estructura encontrada dentro de las cavidades estudiadas reúne las condiciones necesarias para que exista un escenario de fusión-fisión, debido a que la membresía de los individuos cambia continuamente, pero la estabilidad de la colonia se mantiene a largo plazo, como ocurre con otras especies que son más estables en sus grupos sociales (Kerth y König, 1999; Kerth et al., 2001; Willis y Brigham, 2004; Metheny et al., 2008; Popa-Lisseanu et al., 2008). El continuo intercambio entre los miembros de las cavidades hace posible que se establezcan diferentes grados de asociaciones interactivas entre los murciélagos, debido principalmente, como se observó, a su bajo grado de cohesividad. En sociedades de murciélagos más complejas, el grado de lealtad a un sitio de percha es muy alto, según varios ejemplos que se presentan en el trabajo de Kerth et al.(2006).

Nyctinomops laticaudatus muestra que hay murciélagos que cambian continuamente su sitio de percha. En este estudio los cambios entre los diferentes sitios de percha fueron documentados de manera evidente. Las incorporaciones de nuevos individuos no fueron documentadas en este estudio, pero es obvio que son pocos los individuos de la especie que perchan en forma solitaria. En algunas especies de murciélagos, la falta de consistencia para mantener un determinado grupo o sitio de percha está dada por una baja estructura familiar de sus miembros (Wilkinson, 1987; Kerth et al., 2003; Kerth 2008); en este caso, sería conveniente realizar un estudio de las relaciones de parentesco para determinar el grado de selección familiar que puede existir en la colonia.

La proporción sexual no estuvo sesgada hacia alguno de los sexos, lo cual sugiere que las oportunidades de reproducción están uniformemente distribuidas para todos los miembros de la colonia y que el sistema de apareamiento debe ser mejor estudiado mediante observaciones sistemáticas de la colonia. La proporción sexual fue de 1:1 entre los adultos, sugiriendo una tasa de nacimiento similar para ambos sexos.

Se observó que machos y hembras copulan con diversos individuos, sean de su grupo original o de otro. El número de compañeros sexuales observados durante una misma época, sugiere que el sistema de apareamiento es de tipo promiscuo, como se ha registrado en diferentes ocasiones para diferentes especies de murciélagos (WaiPing y Fenton, 1988; Wilkinson y McCracken, 2003; Kerth y Morf, 2004; Vonhof, et al., 2004; Pitnick et al., 2006). Las cópulas no fueron de tipo violento, como se han registrado en otros murciélagos (Keeley y Keeley, 2004); por el contrario, fueron pasivas, los machos no separan a las hembras del grupo y éstas, a su vez, no muestran ninguna resistencia física.

No se encontró diferencia en peso y tamaño entre machos y hembras, por lo que es probable que la selección sexual no sea una fuerza que esté actuando en la colonia. En muchas especies de murciélagos se ha documentado que la selección sexual determina un marcado dimorfismo entre los 2 sexos (Ortega y Arita, 2000; Voigt et al., 2005; Pitnick et al., 2006).

En el murciélago brasileño de cola libre (T. brasiliensis) se han registrado grupos mixtos de ambos sexos en los 
sitios de percha durante largos periodos de observación (Keeley y Keeley, 2004). En contraste, las especies de murciélagos filostómidos forman estructuras sociales más complejas y éstas permanecen por largos periodos de tiempo. En la colonia de estudio, la formación de nuevos grupos con diferentes miembros fue una actividad común; la membresía se renovaba continuamente con nuevos murciélagos, pero se mantenía la composición mixta de ambos sexos.

Los machos mostraron testículos escrotados durante un periodo breve. Diversos autores establecen una temporada reproductiva precisa para la especie enYucatán (Bowles, 1973; Bowles et al., 1990; Jones et al., 1973). Los datos obtenidos en este estudio corroboran este patrón: los machos mostraron signos reproductivos sólo una parte del año, y un número grande de cópulas únicamente se observó una parte del año (julio con respecto al resto de los otros muestreos). En la visita siguiente, después de las cópulas observadas se registró la presencia de agrupaciones de recién nacidos en las paredes del edificio, similares a las de otras especies de molósidos (McCracken y Gustin, 1991).

Nyctinomops laticaudatus pasa gran parte del día en periodo de descanso. En general, las visitas que realizan los diferentes murciélagos a una cavidad en particular no mostraron signos de disturbio; de igual forma, algunos murciélagos utilizan las grietas de las piedras para moverse entre cavidades sin que eso afecte al grupo. Las visitas fueron indistintas para ambos sexos y no se encontró una tendencia a realizar más visitas por parte de un grupo en particular, como suele ocurrir en algunos filostómidos (Ortega y Arita, 2000).

Se observó que los individuos de $N$. laticaudatus realizan una conducta de olfateo cuando algún individuo llega a la cavidad. Para muchas especies, el olfateo entre los miembros de un grupo es señal de reconocimiento entre sitios o entre compañeros e inclusive permite la discriminación de determinado sexo (Keeley y Keeley, 2004; Nielsen et al., 2006). No se registraron conflictos o interacciones agonísticas durante la época de reproducción, que es cuando potencialmente los individuos pueden tener un mayor conflicto entre ellos. En algunas especies, las interacciones agonísticas son muy marcadas, especialmente cuando las hembras se encuentran receptivas (McCracken y Bradbury, 1981; Williams, 1986; Ortega y Arita, 2000; Muñoz-Romo et al., 2008). De igual forma, se documentó que las hembras tienden a moverse menos durante la época reproductiva, lo cual suele ser común cuando que se encuentran en periodo de lactancia, y proporciona un poco de estabilidad a los grupos,(McCracken y Gustin, 1991). Probablemente, las hembras no realizan tantos movimientos entre sitios de percha durante la lactancia porque se encuentran haciéndose cargo de los recién nacidos. El corto periodo de estabilidad termina cuando los recién nacidos aprenden a volar y se incorporan a las cavidades.

El presente estudio describe la estructura social y la composición temporal de una colonia de $N$. laticaudatus. Para entender la forma de estructuración de esta sociedad de murciélagos son necesarios más estudios, sobre todo de índole genética, que permitan conocer los lazos familiares que existen en la colonia, así como la dinámica del sistema de apareamiento y de adquisición de pareja. De igual manera deben estudiarse los beneficios mutuos de una asociación mixta, los cuales necesitan elucidarse, ya que todavía no se han comprendido por completo.

\section{Agradecimientos}

Los comentarios de K. Stoner y E. Vázquez fueron valiosos durante la realización de este estudio. T. Wright nos proveyó con una clara revisión al manuscrito e hizo comentarios muy certeros al mismo. El segundo autor tuvo una beca CONACYT durante el estudio (203300CONACYT). Nuestro más sincero agradecimiento por su invaluable asistencia a R. Cruz, E. Vázquez, S. CastañedaRico, D. Navarrete, G. Rodríguez-Tapia, F. Villalobos y G. Castellanos. Los permisos para realizar nuestras observaciones en el área de estudio fueron dados por el Centro INAH-Yucatán por intermedio de M. F. Sodi Miranda y de J. Huchim.

\section{Literatura citada}

Anthony, E. L. 1988. Age determination in bats. In Ecological and behavioral methods for the study of bats, T. H. Kunz (ed.). Smithsonian Institution Press, Washington, D. C. p. 47-58.

Ávila-Flores, R., J. J. Flores-Martínez y J. Ortega. 2002. Nyctinomops laticaudatus. Mammalian Species 697:1-6.

Balmori, A. 2003. Avances en el conocimiento de la biología y organización social del murciélago rabudo (Tadarida teniotis). Galemys 15:37-53.

Bohn, K. M., G. S. Wilkinson y C. F. Moss. 2007. Discrimination of infant isolation calls by female greater spear-nosed bats, Phyllostomus hastatus. Animal Behaviour 73:423-432.

Bowles, J. B. 1973. Notes on reproduction in four species of bats from Yucatan, Mexico. Transactions of the Kansas Academy of Sciences 75:271-272.

Bowles, J. B., P. D. Heideman y K. R. Erickson. 1990. Observations on six species of free-tailed bats (Molossidae) from Yucatan, Mexico. Southwestern Naturalist 35:151157.

Brooke, A. P. 1997. Social organization and foraging behaviour of the fishing bat, Noctilio leporinus (Chiroptera: Noctilionidae). 
Ethology 103:421-436.

Bouchard, S. 2001. Sex discrimination and roostmate recognition by olfactory cues in the African bats, Mops condylurus and Chaerephon pumilus (Chiroptera: Molossidae). Journal of Zoology 254:109-117.

Chaverri, G., O. E. Quiros, M. Gamba-Ríos y T. H. Kunz. 2007. Ecological correlates of roost fidelity in the tent-making bat Artibeus watsoni. Ethology 113:598-605.

Davidson, S.M., y G. S. Wilkinson. 2004. Function of male song in the greater white-lined bat, Saccopteryx bilineata. Animal Behaviour 67:883-891.

Dechmann, D. K. N., E. K. V. Kalko y G. Kerth. 2007. Alloffspring dispersal in a tropical mammal with resource defense polygyny. Behavioral Ecology and Sociobiology 61:1219-1228.

Jones, J. K., JR., J. D. Smith y H. H. Genoways. 1973. Annotated checklist of mammals of the Yucatan Peninsula, Mexico. I. Chiroptera. Occasional Papers of The Museum, Texas Tech University 13:1-31.

Keeley, A. T. H., y B. W. Keeley. 2004. The mating system of Tadarida brasiliensis (Chiroptera: Molossidae) in a large highway bridge colony. Journal of Mammalogy 85:113119.

Kerth, G. 2008. Causes and consequences of sociability on bats. Bioscience 58:737-746.

Kerth, G., y B. König. 1999. Fission, fusion and nonrandom associations in female Bechstein's bat (Myotis bechsteinii). Behaviour 136:1187-1202.

Kerth, G., M. Wagner y B. König. 2001. Roosting together, foraging apart: information transfer about food is unlikely to explain sociality in female Bechstein's bats (Myotis bechsteinii). Behavioral Ecology and Sociobiology 50:283291.

Kerth, G., A. Kiefer, C. Trappmann y M. Weishaar. 2003. High gene diversity at swarming sites suggests hot spots for gene flow in the endangered Bechstein's bat. Conservation Genetics 4:491-499.

Kerth, G., y K. Reckardt. 2003. Information transfer about roosts in female Bechstein's bats: an experimental field study. Proceedings of the Royal Society B 270: 511-515.

Kerth, G., y L. Morf. 2004. Behavioural and genetic data suggest that Bechstein's bats predominantly mate outside the breeding habitat. Ethology 110:987-999.

Kerth, G., C. Ebert y C. Schmidtke. 2006. Group decisionmaking in fission-fusion societies: evidence from two field experiments in Bechstein's bats. Proceedings of the Royal Society B 273: 2785-2790.

Krutzsch, P. H., y E. G. Crichton. 2005. Reproductive biology of the male little mastiff bat, Mormopterus planiceps (Chiroptera: Molossidae), in Southeast Australia. American Journal of Anatomy 178:352-368.

Leippert, D., W. Goymann, H. Hofer, G. Marimuthu y J. Balasingh. 2000. Roost-mate communication in adult Indian false vampire bat (Megaderma lyra): an indication of individuality in temporal and spectral pattern. Animal Cognition 3:99-106.

Lewis, S. E. 1995. Roost fidelity of bats: a review. Journal of Mammalogy 76:481-496.
Málaga, A., y B. Villa-R. 1956. Algunas notas acerca de la distribución de los murciélagos de América del Norte relacionados con el problema de la rabia. Anales del Instituto de Biología, Universidad Nacional Autónoma de México 27:529-569.

McCracken, G. F., y J. W. Bradbury. 1981. Social organization and kinship in the polygynous bat, Phyllostomus hastatus. Behavioral Ecology and Sociobiology 8:11-34.

McCracken, G. F., y M. K. Gustin. 1991. Nursing behavior in Mexican free-tailed bat maternity colonies. Ethology 89:305-321.

McCracken, G. F., y G. S. Wilkinson. 2000. Bat mating systems. In Reproductive biology of bats, E. G. Crichton y P. H. Krutzsch (eds.). Academic, London. p. 321-362.

Metheny, J. D., M. C. Kalkounis-Ruepell, C. K. R. Willis, K. A. Kolar y M. R. Brigham. 2008. Genetic relationships between roost-mates in a fission-fusion society of tree-roosting big brown bats (Eptesicus fuscus). Behavioral Ecology and Sociobiology 62:1043-1051.

Muñoz-Romo, M., E. A. Herrera y T. H. Kunz. 2008. Roosting behavior and group stability of the big fruit-eating bat Artibeus lituratus (Chiroptera: Phyllostomidae). Mammalian Biology 73:214-221.

Nielsen, L. T., D. K. Eaton, D. W. Wright y B. Schmidt-French. 2006. Characteristic odors of Tadarida brasiliensis mexicana Chiroptera: Molossidae. Journal of Cave and Karst Studies 68:27-31.

Nogueria, M. R., A. Pol, L. R. Monteiro y A. L. Peracchi. 2008. First record of Miller's mastiff bat, Molossus pretiosus (Mammalia: Chiroptera), from the Brazilian Caatinga. Chiroptera Neotropical 14:346-353.

Ortega, J., y H. T. Arita. 2000. Defence of females by dominant males of Artibeus jamaicensis (Chiroptera: Phyllostomidae). Ethology 106:395-407.

Ortega, J., J. E. Maldonado, G. S. Wilkinson, H. T. Arita y R. C. Fleischer. 2003. Male dominance, paternity, and relatedness in the Jamaican fruit-eating bat (Artibeus jamaicensis). Molecular Ecology 12:2409-2415.

Ortega, J., y J. E. Maldonado. 2006. Female interactions in harem groups of Artibeus jamaicensis. Acta Chiropterologica 8:485-495.

Pitnick, S., K. E. Jones y G. S. Wilkinson. 2006. Mating systems and brain sizes in bats. Proceedings of the Royal Society B 273:719-724.

Popa-Lisseanu, A. G., F. Bontadina, O. Mora y C. Ibáñez. 2008. Highly structured fission-fusion societies in an aerial-hawking, carnivorous bat. Animal Behaviour 75:471-482.

Ratcliffe, J. M., H. M. ter Hofstede, R. Ávila-Flores, M. B. Fenton, G. F. McCracken, S. Biscardi, J. Blasko, E. Gillam, J. Orprecio y G. Spanjer. 2004. Conspecifics influence call design in the Brazilian free-tailed bat, Tadarida brasiliensis. Canadian Journal of Zoology 82:966-971.

Russ, J. M., P. A. Racey y G. Jones. 1998. Intraspecific responses to distress calls of the pipistrelle bat, Pipistrellus pipistrellus. Animal Behaviour 55:705-713.

Russ, J. M., G. Jones, I. J. Mackie y P. A. Racey. 2004. Interspecific responses to distress calls in bats (Chiroptera: 
Vespertilionidae): a function for convergence in call design? Animal Behaviour 67:1005-1014.

Russo, D., L. Cistrone y G. Jones. 2005. Spatial and temporal patterns of roost use by tree-dwelling barbastelle bats Barbastella barbastellus. Ecography 28:769-776.

Rzedowski, J. 1978. Vegetación de México. Limusa, México, D.F. p. 298.

Voigt, C. C., G. Heckel y F. Mayer. 2005. Sexual selection favours small and symmetric males in the polygynous greater sac-winged bat Saccopteryx bilineata (Emballonuridae, Chiroptera). Behavioral Ecology and Sociobiology 57:457464.

Vonhof, M., H. Whitehead y M. B. Fenton. 2004. Analysis of Spix's disc-winged bat association patterns and roosting home ranges reveal a novel social structure among bats. Animal Behaviour 68:507-521.

Wai-Ping, B., y M. B. Fenton. 1988. Nonselective mating in little brown bats (Myotis lucifugus). Journal of Mammalogy
69:641-645.

Wilkinson, G. S. 1987. Altruism and co-operation in bats. In Recent advances in the study of bats, M. B. Fenton, P. A. Racey, and J. M. V. Rayner (eds.). Cambridge University Press. p. 299-323.

Wilkinson, G. S. 1992. Information transfer at evening bat colonies. Animal Behavior 44:501-518.

Wilkinson, G. S., y G. F. McCracken. 2003. Bats and balls: sexual selection and sperm competition in the Chiroptera. In Bat ecology, T. H. Kunz and M. B. Fenton (eds.). University of Chicago Press, Illinois. p. 128-155.

Williams, C. F. 1986. Social organization of the bat Carollia perspicilliata (Chiroptera: Phyllostomidae). Ethology 71:265-282.

Willis, C. K. R., y M. Brigham. 2004. Roost switching, roost sharing and social cohesion: forest-dwelling big brown bats, Eptesicus fuscus, conform to the fission-fusion model. Animal Behaviour 68:495-505. 